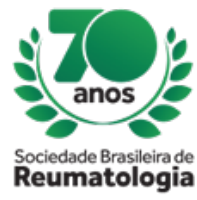

\title{
THE CONSTRUCT VALIDITY OF DIFFERENT KNEE OSTEOARTHRITIS CLASSIFICATION CRITERIA IN RELATION TO BODY MASS INDEX - ELSA-BRASIL MUSCULOSKELETAL (ELSA-BRASIL MSK)
}

Rita de Cássia Corrêa Miguel (Universidade Federal de Minas Gerais, BELO HORIZONTE, MG, Brasil), Rosa Weiss Telles (Universidade Federal de Minas Gerais, BELO HORIZONTE, MG, Brasil), Luciana Andrade Machado (Universidade Federal de Minas Gerais, BELO HORIZONTE, MG, Brasil), Luciana Costa-Silva

(Universidade Federal de Minas Gerais, BELO HORIZONTE, MG, Brasil), Sandhi Maria Barreto (Universidade Federal de Minas Gerais, BELO HORIZONTE, MG, Brasil)

\section{BACKGROUND}

The correct identification of risk factors for knee osteoarthritis (KOA) is important for planning and implementing primary and secondary prevention measures. The great variety of classification criteria used to define KOA makes this investigation difficult, since each criterion emphasizes different characteristics of KOA so identification of these factors depends on the criterion used. Among the risk factors for KOA, obesity is especially important because it can be modified but the literature on this topic still lacks data on the construct validity of KOA classification criteria and obesity, especially in the same population. Objective: to evaluate the aforementioned construct validity by means of the analysis of the strength of association between the body mass index (BMI) and the different criteria.

\section{MATERIALS AND METHODS}

Cross-sectional study of the validation of different KOA classification criteria, namely: Symptomatic ; Radiographic; KOA as defined by the American College of Rheumatology (ACR) and by the National Institute for Health and Care Excellence (NICE), using a subsample of the participants from ELSA-BrasilMSK cohort; an ancillary study of the Longitudinal Study of Adult Health. All the participants were evaluated by a single rheumatologist and only one knee per participant was included. The construct validity was evaluated by investigating the association between BMI and the different criteria and between $\mathrm{BMI}$ and Kellgren-Lawrence $(\mathrm{KL})$ radiographic grade. The associations were tested using logistic regression models (CI95\%; $\alpha=5 \%)$, adjusted for sex and age.

\section{RESULTS}

250 participants were included, (51.2\%), with a mean (SD) age of 56.1 (8.7). BMI was associated with KOA as identified by all the criteria analyzed and the strongest association was found between BMI and the criteria which encompassed radiographic changes (Table 1). It was also found that the strongest association between $\mathrm{BMI}$ and $\mathrm{KL}$ took place at $\mathrm{KL}=4(\mathrm{OR}=1.47 \mathrm{Cl} 95 \%=1.27-1.71)$ but was also present from $\mathrm{KL}=1$.

\section{CONCLUSION}

For studies aiming to investigate obesity and its role in the incidence or progression of KOA, as in studies evaluating the impact of weight loss on individuals with $O A$, we recommend, whenever possible, to classify individuals using criteria that encompass radiographs. 